1 Universidade de Santa Cruz do Sul (Unisc) - Santa Cruz do Sul (RS), Brasil. silva_mendesgabriela@ hotmail.com

2 Universidade de Santa Cruz do Sul (Unisc), Departamento de Enfermagem e Odontologia - Santa Cruz do Sul (RS),

Brasil.

lenid@unisc.br

3 Universidade de Santa Cruz do Sul (Unisc),

Departamento de

Enfermagem e Odontologia

- Santa Cruz do Sul (RS),

Brasil.

mrezende@unisc.br

4 Universidade de Santa Cruz do Sul (Unisc), Grupo de Estudos e Pesquisa em

Saúde - Santa Cruz do Sul (RS), Brasil.

rositelles@gmail.com

5 Universidade de Santa Cruz do Sul (Unisc),

Departamento de

Enfermagem e Odontologia

e Programa de Pós-

Graduação Mestrado

em Promoção da Saúde

- Santa Cruz do Sul (RS),

Brasil.

skrug@unisc.br

\section{Gestão pública no SUS: considerações acerca do PMAQ-AB}

\author{
Public management at the SUS: considerations on the PMAQ-AB
}

\author{
Gabriela Mendes da Silva Flôres', Leni Dias Weigelt'2, Maristela Soares de Rezende, Rosimeri \\ Telles${ }^{4}$, Suzane Beatriz Frantz Krug ${ }^{5}$
}

\begin{abstract}
RESUMO O estudo teve como objetivo investigar as percepções dos gestores municipais de saúde na $28^{\mathrm{a}}$ Região de Saúde do Rio Grande do Sul quanto aos impactos do Programa de Melhoria do Acesso e da Qualidade da Atenção Básica (PMAQ-AB) na organização do trabalho e assistência à saúde, bem como os aspectos que traduzem as percepções e o envolvimento deles com o Programa. Trata-se de uma pesquisa qualitativa com 11 gestores de saúde, com análise de dados a partir da Análise de Conteúdo. Os resultados apresentam aspectos positivos e negativos acerca do PMAQ-AB, evidenciam deficiências no envolvimento dos gestores com o programa, todavia, demonstram impactos positivos para a gestão e assistência em saúde da região em estudo.
\end{abstract}

PALAVRAS-CHAVE Avaliação em saúde. Programas nacionais de saúde. Gestão em saúde.

ABSTRACT The objective of the study was to understand the perception of the municipal health managers in the 28th Health Region of Rio Grande do Sul on the impacts of the National Program for Access and Quality Improvement in Primary Care (PMAQ-AB) in the organization of work and health assistance, as well as the aspects that translate their perceptions and involvement in the Program. It is a qualitative research with 11 health managers and the data were analyzed through Content Analysis. The results show positive and negative aspects of the Program, and detail deficiencies of the managers involvement with it, however the results point to positive impacts on the health management and assistance in the studied region.

KEYWORDS Health evaluation. National health programs. Health management. 


\section{Introdução}

A gestão pública em saúde possui uma forte influência política, devido a isso, desde a Reforma Sanitária Brasileira (RSB), que ocorreu nas décadas de 1970-1980, luta-se pela dignidade humana por intermédio de melhorias na saúde e de políticas que beneficiem a população, por meio da democratização na saúde, a qual deve ser primordial no cotidiano institucional do Sistema Único de Saúde (SUS)'

O cenário da saúde pública brasileira vem sendo moldado por diversas influências sociais, políticas e econômicas vivenciadas ao longo de décadas. Na perspectiva de aprimoramento do SUS, o governo federal, com o intuito de ampliar o acesso e a qualidade na Atenção Primária à Saúde (APS), criou, em 19 de julho de 2011, o Programa Nacional de Melhoria do Acesso e da Qualidade da Atenção Básica (PMAQ-AB), instituído pela Portaria $\mathrm{n}^{\mathrm{o}} 1.654 \mathrm{GM} / \mathrm{MS}$, a fim de alcançar um nível de qualidade semelhante em todas as regiões do País ${ }^{2}$.

O principal objetivo do programa é ampliar o acesso e melhorar a qualidade da atenção básica, fator esse que contribuirá para o desenvolvimento de um padrão de qualidade comparável em todo o País. Além disso, o PMAQ-AB possui o objetivo de satisfazer os usuários, por meio da facilitação do acesso à unidade de saúde e pela qualidade dos serviços prestados. Para isso, o programa objetiva fortalecer a gestão da Atenção Básica (AB), a qual deve promover a autoavaliação e a educação permanente ${ }^{3}$.

O PMAQ-AB possui o intuito de impactar as formas de gestão, trazer uma nova cultura à atenção prestada em saúde e aprimorar a implementação de políticas anteriores com a mesma perspectiva de qualificar a $\mathrm{AB}^{4}$. Ele também contribui financeiramente para municípios que atingem melhorias no padrão de qualidade das equipes da $\mathrm{AB}$.

O programa é dividido por ciclos (hoje se encontra em seu terceiro ciclo, com duração média de 24 meses); fases: fase 1: Adesão e contratualização, fase 2: Certificação (na qual está a avaliação externa, que não deve ser encarada como algo punitivo, mas de reconhecimento do trabalhado desenvolvido pela equipe de $\mathrm{AB}$ e por gestores), fase 3: Recontratualização; e por um conjunto de ações desenvolvidas durante todo o ciclo chamado de Eixo Estratégico Transversal de Desenvolvimento, composto por cinco pilares: autoavaliação; apoio institucional; monitoramento de indicadores de saúde; educação permanente e cooperação horizontal ${ }^{3}$.

Para que o PMAQ-AB seja efetivo nos municípios do Brasil, é necessário, entre outros aspectos, gestão pública de qualidade. Segundo Souza e Viana ${ }^{5}$, gestão em saúde é definida como um processo que cria políticas sanitárias, elege programas que viabilizem tais políticas, além de prover recursos, fiscalizar e avaliar os programas e ações em saúde. A gestão municipal de saúde deve planejar, acompanhar e avaliar todas as ações referentes aos serviços de saúde, possibilitando ações que qualifiquem os serviços, garantindo atuação eficaz do dos gestores ${ }^{6}$.

Sendo assim, o artigo destaca a importância de analisar a visão dos gestores municipais de saúde diante do desafio da melhoria do acesso e da qualidade da atenção básica, já que estes possuem atuação na adesão e desenvolvimento do PMAQ-AB. Ressalta a necessidade de compreensão do papel dos gestores de saúde ante os programas e políticas desenvolvidas no âmbito da APS e do aprimoramento do conhecimento e da assistência por parte dos profissionais de saúde. Por fim, tem o intuito de colaborar para o fortalecimento das diretrizes que norteiam o PMAQ-AB, as quais enfatizam a importância do envolvimento, mobilização e responsabilidade da gestão pública de saúde em todas as fases do programa a fim de alcançar seus propósitos.

Este estudo tem como objetivo investigar as percepções dos gestores municipais 
de saúde sobre o PMAQ-AB na $28^{\mathrm{a}}$ Região de Saúde do Rio Grande do Sul quanto aos impactos na organização do trabalho e na assistência à saúde, bem como os aspectos que traduzem as percepções e o envolvimento desses administradores com o programa.

\section{Metodologia}

Este estudo é um recorte da pesquisa 'Aplicação do Programa Nacional de Melhoria do Acesso e da Qualidade da Atenção Básica (PMAQ-AB): um olhar avaliativo dos profissionais de saúde', desenvolvida por pesquisadores do Grupo de Estudo e Pesquisa em Saúde (Geps) da Universidade de Santa Cruz do Sul (Unisc), Rio Grande do Sul. Trata-se de uma pesquisa qualitativa, de caráter exploratório descritivo. Fizeram parte do estudo 11 gestores municipais de saúde (também podendo ser denominados como secretários de saúde), sendo um gestor de cada um dos 11 municípios da $28^{\mathrm{a}}$ Região de Saúde do Rio Grande do Sul, que aceitaram participar da pesquisa. Para critério de inclusão no estudo, o município deveria ter aderido ao PMAQ-AB.

A $28^{\mathrm{a}}$ Região de Saúde do Rio Grande do Sul é composta por 13 municípios, situados na região central do estado, com cerca de 330.098 mil habitantes. Essa região apresenta uma área geográfica heterogênea, com extensas áreas rurais, destacando as áreas da agricultura e da pecuária, sendo o tabaco o principal produto cultivado. Nas áreas urbanas, destacam-se as atividades industriais e de comércio. A região é composta por 22 Unidades Básicas de Saúde, 54 Estratégias Saúde da Família e 9 hospitais gerais, 13 secretarias municipais de saúde das quais 11 aderiram ao PMAQ-AB7 .

A coleta de dados ocorreu nos locais de trabalho de cada gestor municipal de saúde, após contato prévio. Os dados foram coletados mediante entrevistas semiestruturadas gravadas, com questões norteadoras.
Para analisar os dados, utilizou-se a técnica de Análise de Conteúdo, na modalidade Análise Temática ${ }^{8}$. Na primeira fase, de pré-análise, organizaram-se os dados, iniciando com leituras sucessivas. Na segunda fase, de exploração do material, realizaram-se a codificação e a enumeração dos dados, na qual criaram-se três categorias de análise: Percepções acerca do PMAQ-AB; Impactos do PMAQ-AB na organização do trabalho e na assistência em saúde; Envolvimento dos gestores municipais de saúde com o programa. Na terceira fase, de tratamento dos resultados, inferência e interpretação dos dados coletados, as falas foram organizadas conforme as categorias elaboradas na fase anterior. Após, os resultados foram analisados e interpretados a partir de embasamentos teóricos relacionados com o tema.

Os princípios éticos desta pesquisa foram fundamentados pela Resolução $\mathrm{n}^{\mathrm{o}} 466$, de 12 de dezembro de 2012, do Conselho Nacional de Saúde (CNS), a qual dispõe sobre pesquisas realizadas com seres humanos9. O presente estudo foi aprovado pelo Comitê de Ética em Pesquisa da Universidade de Santa Cruz do Sul sob protocolo 1.171.974/15. Para manter o anonimato dos sujeitos, classificaram-se as entrevistas em Entrevistado Gestor (EG), seguido de ordem numérica.

\section{Resultados e discussão}

Dos 11 gestores municipais de saúde participantes do estudo, 6 eram mulheres, com idades variando de 30 a 68 anos, prevalecendo a faixa etária dos 40 aos 50 anos. Entre os entrevistados, 8 eram casados. Para Grossmann e Nunes ${ }^{10}$, a participação feminina possui a competência de humanizar a gestão, a fim de alcançar a dignidade da pessoa humana. Assim, tal predominância do sexo feminino entre os gestores pode demonstrar o empoderamento feminino em relação aos cargos de 
gestão e/ou políticos do País.

No que diz respeito à escolaridade, oito sujeitos possuem ensino superior completo, um possui ensino superior incompleto e dois possuem ensino médio completo. As formações de nível superior são na área de administração (4), fisioterapia (1), medicina (3). Cinco gestores possuem especialização na área de gestão, sendo quatro na área da saúde. Embora o cargo de gestor municipal de saúde seja um cargo político, é importante que tal profissional possua conhecimento técnico-científico na área. Percebe-se que a minoria dos gestores possui graduação na área de saúde, o que pode comprometer o conhecimento e envolvimento deles no planejamento e desenvolvimento das ações em saúde.

O tempo de trabalho variou de menos de 1 ano ( 3 sujeitos), de 1 a 3 anos ( 2 sujeitos) e de 4 a 6 anos (6 sujeitos). Por não ser cargo de carreira, a rotatividade desses profissionais é inevitável, o que pode dificultar a continuidade das ações em saúde, para isso é necessário um planejamento conciso das ações e grande envolvimento de todos os profissionais de saúde.

Todos os gestores residem no município em que atuam. Oito dos gestores cumprem 40 horas semanais, um gestor 35 horas semanais, um gestor cumpre 44 horas semanais e um não tem horário fixo de trabalho.

\section{O PMAQ-AB na percepção dos ges- tores municipais de saúde}

Nesta categoria, são abordadas as concepções a respeito do programa, sob a ótica dos gestores municipais de saúde. A maioria deles relata percepções positivas acerca do PMAQ-AB, sem, no entanto, especificá-las. Entre os que apontaram percepções positivas, destaca-se, principalmente, o potencial do programa na motivação dos trabalhadores decorrente do incentivo financeiro que este proporciona, de acordo com o desempenho da equipe:
Eu acho bem interessante, [...] é alguma coisa assim para o pessoal ter mais vontade de trabaIhar, que como vem o recurso, ás vezes o pessoal fica naquela eu queria fazer tal coisa, mas eu não posso, porque não tenho dinheiro, então assim, é muito interessante, como município, como gestor a gente fica bem tranquilo com essa questão do PMAQ. (EG4).

Barreto $^{11}$ aborda que o recurso financeiro pode ser uma importante ferramenta motivacional, evidenciando que esse método é mais eficaz em ações pontuais e em mudanças de curto prazo, portanto, em ações mais complexas, que precisam de avaliações frequentes e de um monitoramento eficiente das ações. É necessário que os gestores desenvolvam um planejamento de qualidade para aprimorar as ações do PMAQ-AB e o recurso financeiro possa ser mais um motivador aos profissionais para desempenhar um trabalho eficiente na busca pela qualificação da $\mathrm{AB}$.

Percebeu-se ainda que o PMAQ-AB é considerado pelos gestores como uma importante ferramenta de avaliação das ações em saúde, capaz de proporcionar à gestão um olhar crítico sobre elas:

Eu acho que é bom, por que ás vezes a gente não consegue fazer a avaliação, e assim tu podes avaliar a rede como um todo desde o que o usuário está sentindo e como a gente está oferecendo o serviço, é bem importante. (EG1).

A avaliação tem se tornado essencial para a gestão a fim de que ocorram mudanças efetivas na atenção em saúde. É preciso avaliar as ações realizadas com um olhar ampliado, construindo reflexões acerca dos nós críticos encontrados e sistematizando as ações a serem implementadas. Dessa forma, a avaliação possui grande potencial na identificação dos problemas, na organização das ações, a fim de possibilitar bons resultados na gestão em saúde ${ }^{12}$.

Segundo Cruz et $a .^{13}$, mais de $82 \%$ das 
equipes de $\mathrm{AB}$ das regiões Nordeste, Sudeste e Sul fazem uso do instrumento de Autoavaliação para Melhoria do Acesso e da Qualidade da Atenção Básica (AMAQ) para a realização da autoavaliação, porém, a grande maioria das equipes interpreta as ações de planejamento e de avaliação como rotina burocrática e não como ferramenta potencial de mudanças. Tal interpretação inviabiliza a eficácia dos serviços, pois, a qualificação das equipes da $\mathrm{AB}$ ocorre por meio da percepção da relação entre a avaliação e o planejamento das ações, sendo possível a construção das ações em saúde de forma não fragmentada.

Por outro lado, foram citadas percepções negativas acerca do PMAQ-AB devido ao desconhecimento de aspectos do programa, o que leva os gestores a se sentirem pouco instrumentalizados:

Eu acho que os instrumentos assim que é dado é razoável, [...] a gente trabalha mais ou menos sozinha, porque eles vêm uma vez, como o governo não está perto a gente que resolve aplicar dessa maneira. (EG7).

A gestão municipal possui um importante papel como disparador do apoio institucional às equipes de saúde da $\mathrm{AB}$, mas muitas delas encontram-se desassistidas, colocando em risco a eficácia do programa. Tal situação foi percebida em 2014 no estudo de Melo et al. ${ }^{14}$ quando a maioria dos gestores da $\mathrm{AB}$ das capitais brasileiras encontrava dificuldades em prestar apoio institucional às equipes. Isso evidenciou, segundo os autores do estudo, a necessidade de maior apoio das demais esferas de gestão por intermédio da estruturação da gestão municipal com táticas que buscassem ultrapassar o caráter normativo e de incentivo financeiro.

Tal situação pode remeter a deficiências na comunicação e articulação entre a gestão municipal de saúde e os demais entes federativos. Segundo o Manual Instrutivo $3^{\circ}$ Ciclo (2015 - 2016)3 , o PMAQ-AB aborda o apoio institucional como um compromisso federal, estadual e municipal, a fim de potencializar as ações na qualificação da atenção básica; este apoio é fundamental para transformar a gestão verticalizada em uma gestão horizontal na qual fortalece as relações, considerando as demandas de cada localidade.

\section{Participação e envolvimento com o programa}

Nesta categoria de análise, abordam-se aspectos que traduzem estratégias e ações de envolvimento dos gestores municipais de saúde com o programa. De forma geral, percebe-se que tal envolvimento ocorre por meio de reuniões com as equipes da $A B$ a fim de apresentar o programa, suas fases e as ações a serem desenvolvidas. Além disso, os gestores atuam mais precisamente no gerenciamento do programa, por meio do controle das ações, avaliação das equipes e resultados a serem atingidos:

[...] a gente tem uma participação indireta no processo, a gente deixa mais a equipe que tá lá no trabalho e de acordo com os recursos que a gente recebe do $P M A Q$ que é esse programa de melhoria de qualidade do acesso e a gente desenvolve ações, mas baseadas nas informações que a gente recebe. Então a participação realmente do gestor no processo é uma participação indireta de controle, não é? E dar liberdade ampla para que eles desenvolvam os trabalhos necessários para melhorar o acesso e a qualidade. (EG6).

No estudo de Feitosa et al. ${ }^{15}$, os profissionais e gestores de Grossos, Rio Grande do Norte, apontam que as potenciais mudanças provocadas pelo programa só são alcançadas por meio do envolvimento de todos os profissionais e gestores em saúde em todas as fases do programa.

Mesmo que a participação seja indireta, como relata EG6, é necessário que o gestor faça parte dos processos de desenvolvimento do programa, pois sua participação efetiva 
fortalece os mecanismos de articulação, a fim de que os resultados do programa provoquem mudanças efetivas nas equipes, nas unidades de saúde e atenção à saúde da população. Por outro lado, alguns gestores relatam não ter se envolvido em todas as fases do programa, sendo que a participação se deteve mais na adesão e no acesso ao portal do gestor:

Não, nada. Eu até acessei esses dias, porque tinha que acessar, pedia um acesso, daí eu respondi, fiz uma resposta vaga, porque precisava de uma resposta, então eu acessei o programa, uma vez só, ali precisava porque era uma prévia, mas muito vaga porque eu precisava fazer isso até tal dia, mas não recebi nenhum tipo de orientação também. (EG2).

É observado nessa fala certo desconhecimento das ações do programa, o que dificulta o seu envolvimento de forma mais efetiva em todas as fases do programa, e não apenas na sua adesão. Diante dessa situação, é importante salientar que somente 4 dos 11 gestores possuem graduação na área da saúde, o que pode ser um importante fator dificultador na condução das ações do programa.

Para os gestores, a falta de orientações e a pouca vivência em saúde pública dificultam esse envolvimento com o programa, tal situação não traduz uma situação isolada, sendo essa percebida em outros estudos, como o de Gomes et al. ${ }^{16}$, para o qual o despreparo técnico dos gestores e a alta rotatividade deles influenciam diretamente no planejamento e desempenho das ações.

Por outro lado, identifica-se que a deficiência no envolvimento dos gestores nas diversas fases do programa também decorre do curto período de permanência deles à frente da gestão em saúde:

Tô esperando, não participei aqui ainda, não peguei a fase do PMAQ aqui [...], faz pouco tempo que estou como secretária, minha vivência era só auditoria, mas longe da atenção básica. (EG13).
As frequentes substituições de gestores municipais de saúdes, pode comprometer a execução do programa.

[...] teve momentos que houve uma lacuna nessa minha caminhada, que eu saí da secretaria e era outro gestor [...] na época (da adesão) o gestor era a fulana e depois a fulana né, depois quando eu voltei pra cá quem estava coordenando este trabalho era a enfermeira fulana, não está mais conosco, era um cargo de confiança que nós tínhamos também do governo, com ela que eu conversei mais incisivamente pra ver já os resultados finais, aí ficou um pouco abaixo do esperado da nossa expectativa também. (EG10).

O estudo de Lopes, Scherer e Costa ${ }^{17}$ corrobora tal situação aqui mencionada, pois eles abordam a descontinuidade do desenvolvimento de melhorias no processo de trabalho devido à transferência de profissionais da equipe de saúde, o que interfere diretamente nos resultados do programa.

Tal cenário é um dos desafios a serem enfrentados pelo programa, que requer maior envolvimento dos gestores municipais. Todavia, entende-se que a descontinuidade das ações em saúde, em decorrência das repetidas substituições dos gestores municipais, contribui para que a gestão municipal do programa seja desenvolvida em parceria com outros profissionais.

Em algumas situações, entretanto, percebe-se que tal relação compromete o envolvimento do gestor em todas as fases do programa:

Olha o que eu vou te dizer, na realidade como eu comecei agora eu estou com a menina do administrativo que ela cuida diretamente disso, eu estou mais no gerenciamento geral, mas o PMAQ tá mais direto com ela, até seria interessante tu conversar com ela também. (EG5).

Essa situação traduz a fragmentação das ações em saúde, colocando em risco o andamento dos processos de qualificação da $\mathrm{AB}$. 
Para Arendt ${ }^{18}$, o fato de gerir e coordenar as ações em saúde não presume que o gestor centralize o poder da decisão unicamente em si.

Por intermédio do PMAQ-AB, busca-se uma gestão horizontal, saindo do modelo verticalizado, no qual as ações não devem ser exercidas unicamente pelo gestor, mas também por todos os profissionais de saúde, pois o empenho e engajamento de todos influenciam diretamente nelas. Portanto, a busca por uma gestão horizontal não exime o gestor de suas responsabilidades, pois seu envolvimento com os programas e ações em saúde é essencial para a eficácia dos processos.

Por outro lado, percebe-se na fala de alguns entrevistados que tais substituições não são obstáculos para o envolvimento do gestor com o programa.

[...] a gente está fazendo reuniões semanais pra mim poder me inteirar de todos os assuntos, pra mim tentar ver o que foi pedido na última vez, [...] eu me inteirei do assunto, vi o que precisa, o que o PMAQ faz, qual é o tipo de avaliação, o que vai precisar, o que precisa melhorar. Essa autoavaliação a gente tem que ter no primeiro momento que é o $A M A Q$, isso daí a gente tá fazendo [...]. Então eu não participei de nenhuma das outras fases, gora que eu estou participando. (EG4).

Tal cenário revela a mobilização dos gestores provocada pelo programa. A busca pelo conhecimento do desempenho do programa e a interação entre gestores e profissionais de saúde fortalecem os processos de mudança na AB. Essa interação foi percebida no estudo de Gomes et al. $^{16}$, que analisa a prática da gestão em um município do Espírito Santo, no qual se percebeu que muitas gestões sofrem interferências externas, como: influências políticas, rotatividade de gestores, deficit de capacitações, entre outras. Contudo, há gestores que buscam trabalhar em equipe com a formação do vínculo gestor-equipe de saúde.

\section{Impactos do PMAQ-AB na organi- zação do trabalho e assistência em saúde}

Nesta categoria, é abordada a visão dos gestores municipais de saúde sobre os impactos do PMAQ-AB na organização do trabalho das equipes de $\mathrm{AB}$ e na assistência à saúde da população. Todos os gestores apontaram que o programa provocou impactos positivos, já que, para a grande maioria dos entrevistados, o programa contribuiu para melhorias nas estruturas físicas das unidades e possibilitou a aquisição de novos materiais e equipamentos. Tais mudanças têm auxiliado na acessibilidade para os usuários e profissionais e no desempenho das ações em saúde: "[...] até essa rampa de acesso, que não tínhamos acessibilidade nesse prédio, foi utilizado com esse recurso" (EG8).

[...] equipamentos né, uma coisa a mais que se possa substituir, as vezes que tem que se comprar novo daí se usa esse recurso, a gente substitui frequentemente esses materiais das unidades os estetos, esfigmos, se precisam de substituição também é utilizado parte desse recurso, como ela disse, daí pode colocar um Split numa unidade, em algum lugar que não tenha, a gente usa desse recurso, não é muito mas dá pra alguma coisa. (EG11).

Percebe-se que mudanças nas estruturas físicas contribuem para a qualidade de acessibilidade dos usuários dos serviços, os quais podem ser acolhidos na unidade independentemente de suas limitações físicas. Tal impacto não contribui apenas para o bem-estar físico das pessoas, mas também para a dignidade e cidadania delas, contribuindo para o desempenho das ações realizadas pelos trabalhadores. Para Lopes, Scherer e Costa ${ }^{17}$, as condições de estrutura física e materiais implicam na resolutividade das ações, o que influencia diretamente na qualificação da $A B$.

Além disso, para os gestores, a implantação 
da prática do acolhimento foi um dos grandes impactos do PMAQ-AB. Tal fato reflete diretamente na assistência à saúde mediante mudanças na organização do trabalho, contribuindo para melhorias no acesso dos usuários ao serviço.

[...] nós tivemos a exclusão das fichas, então passamos a trabalhar com classificação de risco, triagem a prontuário eletrônico, [...], eles tiveram tipo um 'baque' assim, muita coisa eles achavam impossível assim de realizar. Influenciou até na motivação da equipe eu acredito, porque modernizou muito, modernizou o serviço com certeza. (EG3).

Segundo Medrado, Casanova e Oliveira'19, o acolhimento facilita o acesso dos usuários à $\mathrm{AB}$, sendo fundamental na organização dos processos de trabalho e nos cuidados com os pacientes. Em estudo realizado pelos autores com profissionais e gestores da saúde em regiões do estado do Rio de Janeiro, o acolhimento foi apontado como uma das principais mudanças no processo de trabalho a partir da adesão ao PMAQ-AB, evidenciando que a grande maioria das equipes trabalha com esta ferramenta. Tal resultado se assemelha com o presente estudo no qual o acolhimento, segundo os entrevistados, é um dos principais resultados positivos da adesão ao PMAQ-AB, sendo evidenciado em todos os municípios que aderiram ao programa na $28^{\text {a }}$ Região de Saúde.

Outro impacto positivo, identificado por alguns gestores, diz respeito as estratégias de qualificação dos profissionais, garantindo a eficácia das ações em saúde:

[...] quando eles têm que fazer uma qualificação, alguma coisa que eles trazem que tira de área, um curso, a gente ajuda eles, porque é bem interessante né, eu acho que o profissional tem que se qualificar, ele não pode ficar parado no tempo, todo ano tem coisa nova, está sempre surgindo coisa nova, então ele tem que se qualificar e ver o que é melhor. (EG4).
Este resultado positivo do programa foi observado também no estudo de Machado et al. ${ }^{20}$, no qual é evidenciado, por meio da avaliação do PMAQ-AB, o desenvolvimento de práticas educativas entre os profissionais da saúde de Mato Grosso do Sul, sendo estas desenvolvidas na maioria das equipes de saúde do estado. Por outro lado, é importante ressaltar o estudo de Lopes, Scherer e Costa ${ }^{17}$, que aborda a existência de práticas de educação permanente que não correspondem às necessidades locais das equipes de $\mathrm{AB}$, o que acaba colocando em risco as transformações das práticas em saúde e a eficácia delas.

A ampliação das ações das Estratégias Saúde da Família também foi evidenciada por alguns gestores como impacto do programa na assistência à saúde, pois possuem o intuito de ir ao encontro das realidades sociais e das necessidades da população local:

[...] há um plano de melhora através de oficinas terapêuticas que eles também fazem junto à comunidade, e então enfim, eles conseguem lá depois direcionar as melhorias que estão sendo feitas. (EG6).

Lopes, Scherer e Costa ${ }^{17}$ também apontam em seu estudo mudanças nas práticas de promoção à saúde, como a formação de grupos e a criação de vínculo entre equipe de saúde e comunidade. Tais mudanças vão ao encontro dos princípios da $\mathrm{AB}$, para os quais, segundo o Manual Instrutivo $3^{\circ}$ Ciclo $(2015-2016)^{3}$, é preciso compreender o usuário na sua singularidade, considerando seus aspectos socioculturais, a fim de promover a saúde por meio de ações integrais.

Estudo realizado em Grossos, Rio Grande do Norte, em 2014, por Feitosa et al.15, analisou as mudanças do PMAQ-AB a partir da visão dos profissionais de saúde e de gestores responsáveis pela coordenação do programa. Tal pesquisa evidenciou resultados semelhantes ao presente estudo, pois, segundo os autores, as principais mudanças provocadas pelo programa foram melhorias 
na organização do trabalho, na estrutura física das unidades e disponibilidade de materiais e organização dos registros das ações em saúde. Para os autores, tais mudanças remetem ao alcance do padrão de qualidade estipulado pelo programa e demonstram a contribuição da sua implementação.

\section{Considerações finais}

A gestão pública em saúde é responsável pelo gerenciamento do pleno desenvolvimento das ações em saúde, a fim de que estas sejam efetivas, porém, muitos obstáculos são encontrados, o que acaba gerando fragilidades em tais ações. Nesse sentido, é necessária a busca por mudanças nos processos de trabalhos, na assistência aos usuários e na gestão dos recursos em saúde, a fim de que a $\mathrm{AB}$ seja qualificada e equiparada em todo território nacional. Nesse sentido, o PMAQ-AB tem sido um importante agente de mudanças, com potencial para a melhoria da qualidade dos serviços, dos processos de trabalho e ações em saúde.

Neste estudo, foi possível perceber que o PMAQ-AB provocou mudanças nos aspectos de gestão. Para alguns gestores, o programa tem sido uma importante ferramenta de avaliação dos serviços em saúde, modificando efetivamente a gestão e assistência em saúde. Além disso, o PMAQ-AB é visto como um programa que motiva os profissionais por meio do incentivo financeiro, favorecendo a eficácia das ações.

Quando evidenciado o desconhecimento do programa por parte de alguns gestores, percebem-se deficiências na comunicação entre as três esferas governamentais de gestão em saúde, restringindo mudanças esperadas com o PMAQ-AB, o que impossibilita o pleno desenvolvimento do programa. É importante que a gestão municipal de saúde busque estratégias de comunicação eficientes entres as três esferas de gestão em saúde e informações de programas, projetos e políticas públicas do Ministério da Saúde, os quais gerem incentivo financeiro para a $\mathrm{AB}$ do município.

A participação ativa dos gestores nas ações de saúde do município e a busca pelo conhecimento das políticas públicas de saúde são essenciais para a qualificação da AB; as políticas não são eficazes por si só, é necessário o engajamento de todos os profissionais para que esta gere efeitos nas equipes de saúde e na comunidade. Ficou evidente neste estudo o envolvimento da maioria dos gestores municipais de saúde com o programa, porém, ainda é necessário que alguns deles se apropriem mais dos processos e ações do PMAQ$\mathrm{AB}$, sendo fundamental que a gestão possua um olhar ampliado sobre as ações em saúde na busca dos princípios e diretrizes do SUS. Além disso, é importante que profissionais de saúde contribuam para a gestão dos programas e políticas públicas de saúde, com conhecimento teórico-prático, a fim de que as fragilidades encontradas no envolvimento dos gestores municipais de saúde sejam minimizadas.

O programa possui o intuito de qualificar a $A B$, visto que é possível perceber nas falas dos gestores os impactos por intermédio do recurso financeiro, o que possibilita melhorias nas estruturas físicas das unidades, aquisição de equipamentos, qualificação dos profissionais, além de gerar mudanças nos processos de trabalho evidenciadas com a implementação da prática de acolhimento aos usuários nas unidades de saúde. Percebese que o PMAQ-AB gerou impactos positivos nas gestões municipais da $28^{\mathrm{a}}$ Região de Saúde, o que demonstra a importância do envolvimento dos gestores e dos profissionais na execução do programa.

Pelo exposto, portanto, é necessário o envolvimento de todos os gestores e profissionais de saúde. Nesse sentido, salienta-se que, a partir do presente estudo, é possível perceber considerações positivas e negativas acerca do PMAQ-AB sob a ótica dos gestores, o que contribui para uma reflexão a 
respeito de uma gestão pública no SUS. É importante salientar que este estudo reflete uma realidade local da gestão pública no SUS ante o PMAQ-AB, sendo de relevância a busca por tais aspectos em outras localidades do território nacional, a fim de que o programa seja desenvolvido de acordo com a realidade local de cada região, em prol do fortalecimento dos princípios e diretrizes da $\mathrm{AB}$.

\section{Colaboradores}

A elaboração deste artigo foi realizada pela graduanda em enfermagem Gabriela
Mendes da Silva Flôres, que realizou a concepção e planejamento da pesquisa, coleta de dados, análise e interpretação dos dados, sob orientação da enfermeira, docente do Departamento de Enfermagem e Odontologia e do Mestrado em Promoção da Saúde da Universidade de Santa Cruz do Sul (Unisc), Suzane Beatriz Frantz Krug. Ambas contribuíram significativamente para a elaboração da pesquisa e para a revisão crítica do conteúdo. A aprovação final do manuscrito foi realizada pela banca examinadora, na qual a enfermeira Suzane Beatriz Frantz Krug fez parte.

\section{Referências}

1. Guizardi FL, Cavalcanti FO. A gestão em saúde: nexos entre o cotidiano institucional e a participação política no SUS. Interface Comun. Saúde Educ. [internet]. 2010 Jul-Set [acesso em 2016 nov 3]; 14(34):633-645. Disponível em: http:// www.scielo.br/scielo.php?script=sci_arttext\&pid $=$ S1414-32832010000300013.

2. Brasil. Ministério da Saúde. Programa Nacional de Melhoria do Acesso e da Qualidade da Atenção Básica-PMAQ/Manual Instrutivo [internet]. Brasília, DF: Ministério da Saúde; 2012 [acesso em 2016 ago 25]. Disponível em: http://189.28.128.100/ dab/docs/publicacoes/geral/manual_instrutivo_ pmaq_site.pdf.

3. Brasil. Ministério da Saúde. Programa Nacional de Melhoria do Acesso e da Qualidade da Atenção Básica (PMAQ): Manual Instrutivo $3^{\circ}$ Ciclo (2015 - 2016) [internet]. Brasília, DF: Ministério da
Saúde; 2015 [acesso em 2016 out 17]. Disponível em: http://189.28.128.100/dab/docs/portaldab/documentos/Manual_Instrutivo_3_Ciclo_PMAQ.pdf.

4. Pinto HA, Sousa ANA, Ferla AA. O Programa Nacional de Melhoria do Acesso e da Qualidade da Atenção Básica: várias faces de uma política inovadora. Saúde debate. 2014 Out; 38(esp):358-372.

5. Souza LEPF, Viana ALD. Gestão do SUS: Descentralização, Regionalização e Participação Social. In: Paim JS, Almeida Filho N. Saúde Coletiva: Teoria e Prática. Rio de Janeiro: MedBook; 2014.

6. Brasil. Portaria $\mathrm{n}^{0} \mathbf{2 . 2 0 3}$, de 5 de novembro de 1996 . Norma Operacional Básica - NOB 1/96 do Sistema Único de Saúde (SUS). Diário Oficial da União. 5 Nov 1996. [acesso em 2016 out 14]. Disponível em: http://bvsms.saude.gov.br/bvs/saudelegis/gm/1996/ prt2203_05_11_1996.html. 
7. Datasus. Departamento de Informática do SUS. Estabelecimentos de Saúde [internet]. Brasília, DF: Ministério da Saúde; 2017 [acesso em 2016 out 20]. Disponível em: http://www2.datasus.gov.br/ DATASUS/index.php?area=0204\&id= 6906 .

8. Bardin L. Análise de conteúdo. Lisboa: Edições 70; 2016.

9. Brasil. Resolução $\mathrm{n}^{\circ} 466$, de 12 de dezembro de 2012 [internet]. Dário Oficial da União. 12 Dez 2012. [acesso em 2016 out 20]. Disponível em: http:// bvsms.saude.gov.br/bvs/saudelegis/cns/2013/ res0466_12_12_2012.html.

10. Grossmann LA, Nunes JBA. A importância da participação política das mulheres para a Construção de uma nova cidadania. In: Anais do $11^{\circ}$ Seminário Internacional de demandas sociais e políticas públicas na sociedade contemporânea. Santa Cruz do Sul: Edunisc; 2014.

11. Barreto JOM. Pagamento por desempenho em sistemas e serviços de saúde: uma revisão das melhores evidências disponíveis. Ciênc. Saúde Colet. 2015; 20(5):1497-1514.

12. Santos TC, Ozorio JC, Ferreira AV, et al. PMAQ-AB e os pactos do SUS: função dispositivo ou repetição. In: LB, Barbosa MG, Ferla AA, organizadores. Atenção Básica: olhares a partir do Programa Nacional de Melhoria do Acesso e da Qualidade (PMAQ-AB). Porto Alegre: Rede Unida; 2016, p. 75-99.

13. Cruz MM. Usos do planejamento e autoavaliação nos processos de trabalho das equipes de Saúde da Família na Atenção Básica. Saúde debate. 2014 Out; 38(esp):124-139.
14. Melo LMF, Martiniano CS, Guimarães J, et al. Análises das diretrizes para o apoio institucional das gestões da Atenção Básica das capitais brasileiras. Saúde debate. 2016 Jan-Mar; 40(108):8-22.

15. Feitosa RMM, Paulino AA, Lima Junior JOS, et al. Mudanças ofertadas pelo Programa Nacional de Melhoria do Acesso e da Qualidade da Atenção Básica. Saúde Soc. 2016; 25(3): 821-829.

16. Gomes LB, Barbosa MG, Ferla AA, orgnizadores. Atenção Básica: olhares a partir do Programa Nacional de Melhoria do Acesso e da Qualidade (PMAQ-AB). Porto Alegre: Rede Unida; 2016.

17. Lopes EAA, Scherer MDA, Costa AM. O Programa Nacional de Melhoria do Acesso e da Qualidade da Atenção Básica e a organização dos processos de trabalho. Tempus (Brasília, DF). 2015; 9(2):237-250.

18. Arendt H. A condição humana. 12. ed. Rio de Janeiro: Forense-Universitária; 2014.

19. Medrado JRS, Casanova AO, Oliveira CCM. Estudo avaliativo do processo de trabalho das Equipes de Atenção Básica a partir do PMAQ-AB. Saúde debate. $2015 ; 39(107): 1033-1043$.

20. Machado JFFP, Carli AD, Kodjaoglanian VL, et al. Educação Permanente no cotidiano da Atenção Básica no Mato Grosso do Sul. Saúde debate. 2015; 39(104):102-113.

Recebido em 13/07/2017

Aprovado em 17/01/2018

Conflito de interesses: inexistente

Suporte financeiro: não houve 